OPEN ACCESS

Edited by:

Nelson C. Soares,

University of Cape Town, South Africa

Reviewed by:

Monika J. Adamczyk-Poplawska,

University of Warsaw, Poland

Ana V. Coelho

Instituto de Tecnologia Química e

Biológica-UNL, Portugal

*Correspondence:

Aleksandra E. Sikora

aleksandra.sikora@oregonstate.edu

Specialty section:

This article was submitted to

Microbial Physiology and Metabolism,

a section of the journal

Frontiers in Microbiology

Received: 01 September 2015

Accepted: 12 October 2015

Published: 26 October 2015

Citation:

Baarda BI and Sikora AE (2015) Proteomics of Neisseria gonorrhoeae:

the treasure hunt

for countermeasures against an old disease.

Front. Microbiol. 6:1190.

doi: 10.3389/fmicb.2015.01190

\section{Proteomics of Neisseria}

gonorrhoeae: the treasure hunt for countermeasures against an old disease

\author{
Benjamin I. Baarda and Aleksandra E. Sikora* \\ Department of Pharmaceutical Sciences, College of Pharmacy, Oregon State University, Corvallis, OR, USA
}

Neisseria gonorrhoeae is an exquisitely adapted, strictly human pathogen and the causative agent of the sexually transmitted infection gonorrhea. This ancient human disease remains a serious problem, occurring at high incidence globally and having a major impact on reproductive and neonatal health. N. gonorrhoeae is rapidly evolving into a superbug and no effective vaccine exists to prevent gonococcal infections. Untreated or inadequately treated gonorrhea can lead to severe sequelae, including pelvic inflammatory disease and infertility in women, epididymitis in men, and sightthreatening conjunctivitis in infants born to infected mothers. Therefore, there is an immediate need for accelerated research toward the identification of molecular targets for development of drugs with new mechanisms of action and preventive vaccine(s). Global proteomic approaches are ideally suited to guide these studies. Recent quantitative proteomics (SILAC, ITRAQ, and ICAT) have illuminated the pathways utilized by $N$. gonorrhoeae to adapt to different lifestyles and micro-ecological niches within the host, while comparative 2D SDS-PAGE analysis has been used to elucidate spectinomycin resistance mechanisms. Further, high-throughput examinations of cell envelopes and naturally released membrane vesicles have unveiled the ubiquitous and differentially expressed proteins between temporally and geographically diverse N. gonorrhoeae isolates. This review will focus on these different approaches, emphasizing the role of proteomics in the search for vaccine candidates. Although our knowledge of $N$. gonorrhoeae has been expanded, still far less is known about this bacterium than the closely related $N$. meningitidis, where genomics- and proteomics-driven studies have led to the successful development of vaccines.

\section{Keywords: Neisseria gonorrhoeae, gonorrhea, proteomics, molecular targets, vaccine, drugs, antibiotic resistance, surveillance}

\section{INTRODUCTION}

Gonorrhea is an ancient human disease, with references to its symptoms found in the Old Testament of the Bible (Leviticus 15:1-3). For almost 700 years, it has been known as "the clap," a likely reference to the old Le Clapiers district of Paris where prostitutes were housed. This sexually transmitted disease remains a global scourge today, causing an estimated 106 million new cases worldwide each year WHO (2012). In the United States, gonorrhea is the second most common sexually transmitted disease, with over 300,000 new cases, primarily affecting those between 
20 and 24 years of age, reported to the Centers for Disease Control and Prevention (CDC) annually CDC (2013). The Gramnegative diplococcus Neisseria gonorrhoeae, the gonococcus (GC), is the sole cause of gonorrhea. In men, infections typically present as profuse, localized inflammatory response of the urethra (i.e., urethritis). In contrast, gonorrhea remains asymptomatic in $50-80 \%$ of infected women (Farley et al., 2003; WHO, 2011). Untreated or inadequately treated gonococcal infections can have severe consequences including epididymitis in men and pelvic inflammatory disease and inflammation of the uterus, ovaries and fallopian tubes in women, which can lead to infertility. Neonatal health is also detrimentally affected by GC infection, as this pathogen can cause a sight-threatening conjunctivitis in infants born to infected mothers (Creighton, 2011). Additionally, infection with GC increases the risk of HIV transmission (Tapsall, 2005). Further compounding the difficulty in treating gonorrheal infections, through a number of point mutations, as well as horizontally acquired genes, GC has gained resistance to nearly all antibiotics currently in use (Tapsall et al., 2010; Unemo and Shafer, 2014). The CDC now recommends a combination of ceftriaxone with either doxycycline or azithromycin for empirical gonorrhea treatment (CDC, 2012b); however, treatment failures with ceftriaxone have been verified in Japan, Australia, Sweden, and Slovenia (reviewed in Unemo, 2015). Additionally, GC demonstrates remarkable heterogeneity and strain-to-strain variability, which represent a significant challenge in vaccine development (Zhu et al., 2011; Jerse et al., 2014).

Immediate action is critically needed against gonorrhea before it becomes completely untreatable. In response to this dire possibility, the World Health Organization (WHO) published the "Global Action Plan to Control the Spread and Impact of Antimicrobial Resistance in Neisseria gonorrhoeae" (WHO, 2012), and the CDC (CDC, 2012a), as well as the European Centre for Disease Prevention and Control (ECDC; ECDC, 2012) proposed region-specific response plans. Overall, these proposals stressed the importance of implementing holistic action against gonorrhea, which would encompass early prevention, diagnosis, contact tracing, treatment, and surveillance of antimicrobial resistance and treatment failures.

An ideal method of gonorrhea prevention would be the development of a protective vaccine(s). Indeed, according to model simulations, gonococcal prevalence could be reduced by at least $90 \%$ after 20 years if all 13-year olds were given a non-waning vaccine with $50 \%$ efficacy or a vaccine with $100 \%$ efficacy that wanes after 7.5 years. Further, even with a nonwaning vaccine of $20 \%$ efficacy, as much as a $40 \%$ decrease in prevalence could be anticipated (Craig et al., 2015). In line with the $\mathrm{WHO}, \mathrm{CDC}$, and ECDC call for action, it is a prerequisite that the anti-gonorrhea vaccine and new drug development be made a priority. Different proteomic approaches are exceedingly valuable to accompany the progress of effective new therapeutic interventions by identifying vaccine and drug targets. Herein, we guide through these different approaches in the treasure hunt for countermeasures against gonorrhea, emphasizing the role of proteomics in the search for GC vaccine candidates (Figure 1).

\section{HOW DOES A HEALTH-OBSESSED RESEARCHER EVALUATE AN ENERGY BAR?}

\section{WITH PROTEOMICS}

As genomic approaches, whole-genome sequencing in particular, have become relatively inexpensive and increasingly highthroughput, with short turn around times and great resolution in the past few years, they have grown increasingly useful in basic research and in clinical diagnosis. The Broad Institute has recently released the whole genome sequences of $14 \mathrm{GC}$ clinical isolates in collaboration with the Neisseria gonorrhoeae Group to facilitate research into pathogenesis and genetic determinants of disease states (Neisseria gonorrhoeae Group Sequencing Project, Broad Institute of Harvard and $\mathrm{MIT}^{1}$ ). Additionally, multilocus sequence typing (MLST), which characterizes isolates based on internal fragments of housekeeping gene alleles, has been used to cluster GC patient isolates based on phenotype (Ilina et al., 2010). A database of MLST data for Neisseria species has been established ${ }^{2}$, further facilitating MLST identification and genotypic grouping of GC isolates. Genomic-derived methodologies have identified GC iron-responsive genes (Ducey et al., 2005), the anaerobic stimulon (Isabella and Clark, 2011), as well as gene expression patterns during infection of the lower female genital tract (McClure et al., 2015). In clinical applications, the proliferation of genomic datasets has promoted the development of nucleic acid amplification tests (NAATs), which allow for rapid identification of GC in patient samples without the need for culture (Low et al., 2014).

Ultimately, however, genomics is unable to capture the complete biological complexity present. A combination of different proteomic approaches can greatly complement genomicacquired data by examining the GC protein population in biofilms or upon exposure to relevant host stimuli (Wu et al., 2010; Phillips et al., 2012), proteins associated with drug resistance (Nabu et al., 2014), proteome expression patterns during infection, posttranslational modifications, or by providing information about proteins' subcellular location, structures and protein-protein interactions. The knowledge gained from proteomic studies can be useful for identifying GC in clinical samples (Gudlavalleti et al., 2008; Carannante et al., 2015), evaluating antibiotic resistance, and discovering potential vaccine and drug candidate proteins (Zielke et al., 2014, 2015).

\section{WHEN DO YOU NOT WANT TO RECEIVE APPLAUSE?}

\section{WHEN YOU IDENTIFY "THE CLAP”}

Rapidly identifying GC in clinical isolates is vital to initiate treatment as quickly as possible to prevent the severe consequences of untreated gonorrhea, as well as to limit the spread of antimicrobial resistant strains. NAATs, developed

\footnotetext{
${ }^{1}$ http://www.broadinstitute.org/

${ }^{2}$ http://pubmlst.org/neisseria/
} 


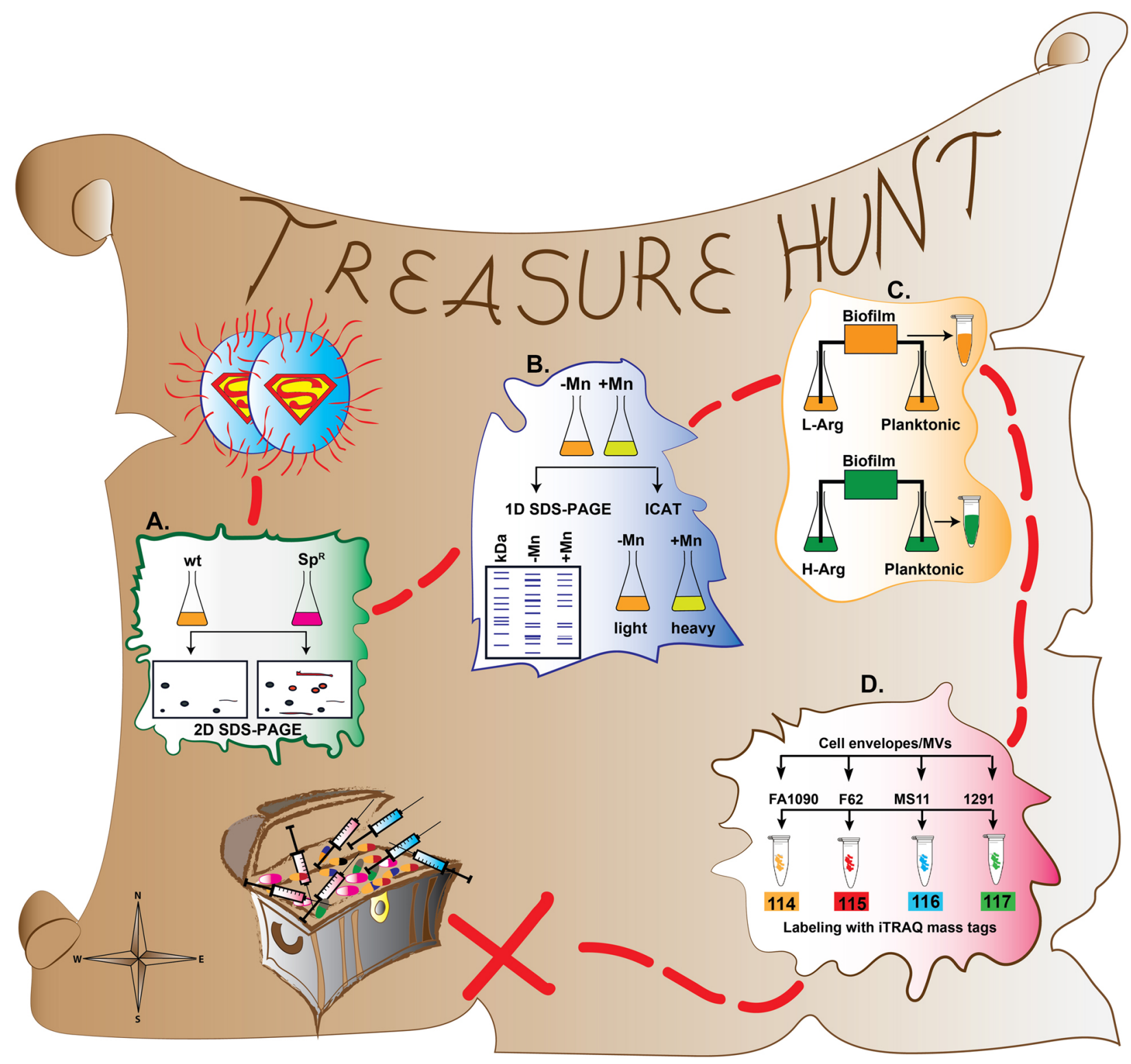

FIGURE 1 | A treasure hunt map of proteomic approaches applied to better understand GC pathophysiology and discovery of vaccine and drug targets. (A) Comparative proteome maps have been constructed between spectinomycin resistant $\left(\mathrm{Sp}^{\mathrm{R}}\right)$ and spectinomycin sensitive (wild type; wt) GC isolates using 2-D-SDS-PAGE. (B) Proteomic investigations (1D SDS-PAGE and ICAT) of manganese (Mn) regulation of virulence factors and oxidative stress. (C) Proteomic profiling of GC transition from planktonic to biofilm growth using SILAC. (D) Comparative, high-throughput proteomic analyses of cell envelope and MV fractions derived from GC strains FA1090, F62, MS11, and 1291.

with the use of genomic data, are more rapid and sensitive than culture, which has resulted in an increase in the number of infections detected (Unemo and Shafer, 2014; Wind et al., 2015). Current commercially available gonococcal NAATs are unable to satisfactorily measure antimicrobial resistance, undermining the surveillance of antimicrobial resistance trends. Because of this limitation, the ECDC has recommended that all specimens positive for GC by NAAT are subsequently cultured to monitor antimicrobial resistance trends (ECDC, 2012). However, laboratory-developed NAATs have been utilized to identify known genetic antimicrobial resistance determinants against several classes of antibiotics as well as detecting the crucial mutations conferring resistance to extended-spectrum cephalosporins in superbug strains H041 and F89 (reviewed, by Unemo, 2015).

As antibiotic resistance determinants continuously evolve, the development of new detection tests is required (Unemo, 2015). Proteomics can provide sensitive, accurate, rapid, and cost-effective methods of GC identification and determination of antimicrobial resistance patterns in clinical samples. Proteomic identification of bacteria is primarily performed with data generated by matrix-assisted laser desorption ionization 
time-of-flight mass spectrometry (MALDI-TOF-MS). Unique and representative biomarker ions can be established from intact cell MALDI-TOF-MS analysis (van Baar, 2000; Amiri-Eliasi and Fenselau, 2001; Fenselau and Demirev, 2001; Fagerquist et al., 2010; Murray, 2010; Niyompanich et al., 2014). The most important advantages in direct bacterial profiling by means of MALDI-TOF-MS are: (1) the requirement for only a small amount of biological material, (2) the possibility of examining intact cells without preceding extraction and separation, (3) a fast and straightforward procedure, and (4) high specificity in species differentiation (Ilina et al., 2009).

One of the first examples of direct GC profiling with MALDITOF-MS used surface enhanced MALDI-TOF-MS to analyze over 350 GC strains and closely related species (Schmid et al., 2005). These comparisons enabled the design of multilayer artificial neural networks that revealed 20 ion peak descriptors of positive, negative and secondary nature that were supreme for GC identification (over $96 \%$ efficiency, a sensitivity of $95.7 \%$ and a specificity of $97.1 \%$ ). Another study used atmospheric pressure MALDI-TOF to determine that a putative DNA binding protein from N. meningitidis; its homolog in GC, DbhA; and acyl carrier proteins in each species could be used as protein biomarkers for identifying pathogenic Neisseria (Gudlavalleti et al., 2008). Although this approach does not provide a method to distinguish between $N$. meningitidis and $N$. gonorrhoeae, it can act as a base upon which to build techniques for identifying pathogenic Neisseria in clinical samples. The successful application of MALDI-TOF-MS for GC identification has also been recently demonstrated on 92 out of 93 isolates of gonococci collected from 2007 to 2012 as part of the European Gonococcal Antimicrobial Surveillance Programme (Carannante et al., 2015).

Together, these studies highlight the potential of proteomic approaches to rapidly and correctly identify GC in various clinical isolates, which, if implemented on a larger scale, will promote rapid initiation of treatment while still allowing antimicrobial susceptibility testing to be performed.

\section{WHAT DID THE BACTERIA CALL THEIR GUERILLA WARFARE UNIT? \\ THE SPECTINOMYCIN RESISTANCE}

In a survey of antimicrobial resistance in Southeast Asia, the WHO Global Gonococcal Antimicrobial Surveillance Program found that between 0.6 and 10.5 percent of isolates demonstrated spectinomycin resistance (Bala et al., 2013). Spectinomycin directly interacts with $16 \mathrm{~S}$ rRNA and inhibits the elongation factor G (EF-G)-catalyzed translocation of the peptidyl-tRNA from the A site to the P site during protein synthesis (Bilgin et al., 1990; Ramakrishnan and White, 1992). Not surprisingly, spectinomycin resistance determinants traditionally involve mutations in $16 \mathrm{~S}$ rRNA (Maness et al., 1974; Galimand et al., 2000). However, a deletion of amino acid 25 and a K26E amino acid alteration (E. coli numbering) in the ribosomal protein S5 is a newly identified mechanism associated with high-level spectinomycin resistance in GC (Unemo et al., 2013). Overall, however, the in vitro susceptibility to this aminocyclitol compound is remarkably high worldwide and this antibiotic is an effective alternative for treatment of anogenital gonorrhea, in particular for multidrug resistant cases (Unemo, 2015).

The proteomic signatures of GC spectinomycin resistance as well as cellular responses to spectinomycin treatment were assessed through qualitative and relative quantitative proteomics using densitometry analysis of proteins separated by two-dimensional sodium dodecyl sulfate-polyacrylamide gel electrophoresis (2D SDS-PAGE) and protein identification with MALDI-TOF-MS (Otto et al., 2012; Nabu et al., 2014). Comparative proteome maps have been constructed between a spectinomycin resistant $\left(\mathrm{Spec}^{\mathrm{R}}\right)$ clinical isolate and a spectinomycin sensitive $\left(\mathrm{Spec}^{\mathrm{S}}\right)$ reference strain (ATCC 49226; Figure 1A). When the two strains were not exposed to spectinomycin, their protein profiles were largely the same, with EF-Tu and EF-Ts, cysteine synthase, and the septum sitedetermining protein $\mathrm{MinD}$ upregulated in the $\mathrm{Spec}^{\mathrm{R}}$ isolate. Additionally, MinD, oxidoreductase, and hypothetical protein NGO1873 were shifted to a more acidic isoelectric point, and the ribosomal protein S6 was shifted to a more basic $\mathrm{pI}$ in the resistant isolate. Finally, ABC transporter substrate-binding protein showed decreased expression and alcohol dehydrogenase was not detected in the $\mathrm{Spec}^{\mathrm{R}}$ strain. Interestingly, in the presence of spectinomycin, although dihydrolipoamide dehydrogenase, peroxiredoxin, an outer membrane protein Rmp, and the $50 \mathrm{~S}$ ribosomal protein L7/L12 were upregulated in both strains, none of the proteins were expressed as highly in the resistant strain as in the wild type. Overall, the spectinomycin treatment of the Spec $S$ GC resulted in alterations in the abundance of proteins involved in energy metabolism and detoxification, as well as cell envelope proteins (Rmp and $\mathrm{ABC}$ transporter substrate-binding protein).

Based on the obtained proteomic comparisons, a mode of spectinomycin action on GC is proposed but not experimentally validated (Nabu et al., 2014). Briefly, in the presence of subminimal inhibitory concentrations, the drug destabilizes the outer membrane, recruiting $\mathrm{Rmp}$ to maintain the integrity of the cell envelope. Spectinomycin accumulates in the periplasm, generating a concentration imbalance between the inside and outside of the cell. In response, significant changes in energy metabolism including an increase in $\mathrm{NADH}$ production and oxidation through the electron transport chain occur. At the same time, additional proton motive force allows more drug molecules to enter the cell. Increased NADH oxidation leads to an increase in reactive oxygen species, which are detoxified by the increase in peroxiredoxin and glutamate dehydrogenase production. Higher levels of L7/L12 allow the cell to overcome the inhibition of ribosomal translocation imposed by spectinomycin. In the $\mathrm{Spec}^{\mathrm{R}}$ strain, amino acid ATP binding cassette transporter substratebinding protein levels are decreased even in the absence of spectinomycin, possibly affecting uptake of the drug. Expression of enolase is decreased, which may result in increased levels of 2P-D-glycerate and 3P-D-glycerate. Together with an increase in cysteine synthetase expression, this may improve the cell's defense against reactive oxygen species. Finally, the already high levels of EF-Tu and -Ts, together with the upregulation of L7/L12, may assist with protein translation in the presence of spectinomycin (Nabu et al., 2014). 
Future research may be able to exploit some of the suggested secondary pathways and increase the efficacy or reduce the resistance potential of future antimicrobial compounds. Also apparent in this study are some of the shortcomings of 2D SDSPAGE, which has limited utility when dealing with low abundance and/or membrane proteins.

\section{HOW DID THE HEALTH-OBSESSED RESEARCHER COMPARE TWO PROTEIN SHAKES?}

\section{WITH QUANTITATIVE PROTEOMICS}

In contrast to qualitative proteomics, quantitative proteomic approaches allow for (absolute or relative) quantification of proteins on a global scale. The object of quantitative proteomics is to identify specific alterations between control samples and particular experimental conditions (e.g., healthy versus diseased state). In addition, quantitative proteomic profiling may focus on a specific subset of proteins (subproteome), where for instance bacterial whole cell lysates are subjected to fractionation to enrich for cell envelope proteins. Very often the proteins of interest are relatively low in abundance; therefore it is critical to utilize appropriate combinations of pre-fractionation techniques such as different kinds of liquid chromatography (affinity, reversed phase, size-exclusion, or ion exchange) to reduce the complexity of analyzed samples, in addition to employing sensitive mass spectrometry instruments. Different approaches have been developed to facilitate quantitative proteome profiling studies involving stable isotope labeling, such as ICAT, ICPL, SILAC, iTRAQ, TMT, and IPTL. In addition, label-free statistical methodologies (MRM, SWATH) and absolute quantification methods by mass spectrometry (AQUA strategy) have become available (reviewed in Chahrour et al., 2015).

Quantitative proteomic approaches, involving different stable isotope labels and summarized in Table 1, have been used to investigate how GC responds to oxidative stress ( $\mathrm{Wu}$ et al., 2010), transitions from planktonic to biofilm growth (Phillips et al., 2012), and adapts the composition of the cell envelope in response to different environmental cues encountered in microecological niches within the host (Zielke et al., 2015). In addition, iTRAQ technology has also revealed the dynamic subproteomes of cell envelopes and naturally released membrane vesicles in four different GC isolates (Zielke et al., 2014).

\section{WHAT HAPPENS WHEN TWO OXEN GO ON THEIR FIRST DATE? OXIDATIVE STRESS}

In a typical inflammatory response to GC, neutrophils are recruited to the site of infection by chemokines, such as IL8 , released by infected mucosal surfaces (Criss et al., 2009). When activated neutrophils phagocytize GC, the production of reactive oxygen species (ROS) is either stimulated or inhibited, depending on GC expression of opacity-associated proteins
(Opa). Cells expressing Opa protein $\left(\mathrm{Opa}^{+}\right)$ligate to human carcinoembryonic antigen-related cell adhesion molecules (CEACAMs), specifically CEACAM3, on the surface of the neutrophil, triggering phagocytosis, NADPH subunit assembly, and degranulation (Schmitter et al., 2007; Sarantis and GrayOwen, 2012). Additionally, Opa 57 protein ligated to CEACAM3 amplifies the inflammatory response by activating nuclear factor (NF) $-\kappa \mathrm{B}$ and increasing phosphorylation of the p38 kinase (Sintsova et al., 2014).

This cascade of ROS production puts the GC cells under tremendous oxidative stress. However, viable GC has been shown to survive and replicate within neutrophils, even after NADPH oxidase activation (Simons et al., 2005), indicating that GC is able to protect itself against oxidative stress. This pathogen can defend against superoxide radicals (Tseng et al., 2001) and hydrogen peroxide (Seib et al., 2004) in a manganese (Mn) dependent manner. A study combining transcriptomic and qualitativeand quantitative-proteomic approaches examined the protective mechanism utilized by GC in the presence of $\mathrm{Mn}$ (Wu et al., 2010). One-dimensional sodium dodecyl sulfate-polyacrylamide gel electrophoresis (1D SDS-PAGE) paired with one-dimensional liquid chromatography-tandem mass spectrometry (1D LCMS/MS) identified 46 proteins expressed only in the presence of $\mathrm{Mn}$. Notably, bacterioferritin, azurin (Laz), and iron-superoxide dismutase (SodB) - proteins involved in defense against oxidative stress-were expressed in bacteria cultured in the presence of $\mathrm{Mn}$, but were not detected in those cultured without Mn. When cultured in the absence of Mn, GC was found to express an outer membrane methionine sulfate reductase that is also involved in defense against superoxide and hydrogen peroxide.

Further, a quantitative proteomic investigation using IsotopeCoded Affinity Tag (ICAT) labeling was used to determine which proteins were differentially regulated by the presence or absence of Mn (Wu et al., 2010). For ICAT analysis, two protein samples are labeled with reactive groups (biotinylated iodoacetamide or acrylamide derivatives) that specifically react with the sulphydryl groups of denatured peptides' cysteine side chains (Gygi et al., 1999). One sample is labeled with a light isotope, while the other sample is labeled with a heavy isotope. The samples are then combined and analyzed by mass spectrometry (Figure 1B; Table 1). The protein populations can then be quantified by comparing the ratio of heavy to light proteins (Gygi et al., 1999; Colangelo and Williams, 2006; Chahrour et al., 2015).

In this study, ICAT labeling, coupled with MS/MS, revealed numerous proteins that were downregulated more than 1.5 -fold in the presence of Mn, including PilT (an ATPase involved in pilus disassembly), OmpR, a $64 \mathrm{KDa}$ outer membrane protein (OMP $\mathrm{P} 64 \mathrm{k})$, and peroxiredoxin, which reduces and detoxifies peroxides (Seib et al., 2006). Additionally, Mn affected the levels of pilin, superoxide dismutase, and pyrophosphatase without causing a corresponding change in the transcript level, indicating that these proteins are likely to be regulated post-transcriptionally by the presence of Mn (Wu et al., 2010).

Taken together, the results of this study suggest that, in the presence of $\mathrm{Mn}, \mathrm{GC}$ upregulates the expression of iron storage proteins that protect against oxidative damage. Concomitantly, the bacterium downregulates pyrophosphatase 
TABLE 1 | Quantitative Proteomic Approaches utilized to studying GC.

\begin{tabular}{|c|c|c|c|}
\hline Proteomic Approach & Principles & Use in GC & References \\
\hline Isotope coded affinity tag (ICAT) & $\begin{array}{l}\text { - Post-harvest labeling } \\
\text { - Heavy or light isotopic reactive groups label cysteine residues in a } \\
\text { protein sample. } \\
\text { - Two samples are pooled and analyzed by MS. } \\
\text { - Relative quantification of proteins by comparing heavy: light ratio. }\end{array}$ & Response to oxidative stress & Wu et al. (2010) \\
\hline $\begin{array}{l}\text { Stable isotope labeling by amino } \\
\text { acids in cell culture (SILAC) }\end{array}$ & $\begin{array}{l}\text { - Metabolic labeling } \\
\text { - Growth medium is supplemented with either light (unlabeled) or } \\
\text { heavy (labeled) amino acid. } \\
\text { - Two samples are pooled and analyzed by MS. } \\
\text { - Relative quantification of proteins by comparing heavy: light ratio }\end{array}$ & $\begin{array}{l}\text { Shift from planktonic to biofilm } \\
\text { growth }\end{array}$ & Phillips et al. (2012) \\
\hline $\begin{array}{l}\text { isobaric Tagging for Relative and } \\
\text { Absolute Quantification (iTRAQ) }\end{array}$ & $\begin{array}{l}\text { - Post-harvest labeling } \\
\text { - Proteolytically digested proteins are tagged on N-termini } \\
\text { and lysine side chains with isobaric mass labels. } \\
\text { - Two to eight samples are pooled and analyzed by MS. } \\
\text { - Relative or absolute quantification of proteins by measuring } \\
\text { intensity of reporter ion peak. }\end{array}$ & $\begin{array}{l}\text { Cell envelopes and membrane } \\
\text { vesicles } \\
\text { Comparison of cell envelope } \\
\text { profiles upon environmental } \\
\text { cues encountered in the host }\end{array}$ & $\begin{array}{l}\text { Zielke et al. (2014); } \\
\text { Zielke et al. (2015) }\end{array}$ \\
\hline
\end{tabular}

(Ppa) and polyphosphate kinase (Ppk). Ppa hydrolyzes inorganic pyrophosphate $\left(\mathrm{PP}_{\mathrm{i}}\right)$ into two molecules of orthophosphate $\left(\mathrm{P}_{\mathrm{i}}\right)$, while Ppk synthesizes polyP from $\mathrm{P}_{\mathrm{i}}$. When these two proteins are downregulated, $\mathrm{PP}_{\mathrm{i}}$ accumulates in the cell and chelates $\mathrm{Mn}$. In a $\mathrm{Mn}(\mathrm{II})-\mathrm{PP}_{\mathrm{i}}$ complex, $\mathrm{Mn}$ is able to defend against ROS non-enzymatically (Wu et al., 2010).

The information gleaned from these proteomic experiments could suggest a method to combat gonorrhea whereby the downregulation of protective proteins required only in the absence of $\mathrm{Mn}$ is maintained, but the alteration of the levels of proteins required for protection from oxidative stress in the presence of $\mathrm{Mn}$ is blocked. Importantly, this study highlights the utility of proteomic approaches to investigate biological responses involving post-transcriptional regulation that genomic methods alone cannot discover. It is important to keep in mind, however, that ICAT has two major drawbacks: (1) proteins that do not have any cysteine residues will be eliminated from this analysis, and (2) the release of biotinylated peptides from the streptavidin column is not quantitative for low-abundance peptides (Chahrour et al., 2015).

\section{WHAT IS A MICROBIOLOGIST'S FAVORITE KIND OF MOVIE?}

\section{A BIOFILM}

Bacteria often shift from planktonic (free living bacteria) growth to a biofilm community, where bacteria grow in close proximity to each other, protected by an extracellular polymer composed of polysaccharides, proteins, nucleic acids, and lipids (Flemming and Wingender, 2010). GC has been shown to form biofilms in vitro (Greiner et al., 2005), and an examination of primary cervical epithelial cells from cervical biopsy samples revealed biofilm growth in culture positive gonorrhea cases (Steichen et al., 2008). Biofilms exacerbate antibiotic resistance by providing a protective barrier against antimicrobial action, and biofilms formed by GC are thought to contribute to asymptomatic infections (Steichen et al., 2008).
To understand the mechanisms underlying biofilm formation, a quantitative proteomic study examined the proteome changes GC undergoes in the transition from planktonic to biofilm growth using stable isotope labeling by amino acids in cell culture (SILAC; Figure 1C; Table 1; Phillips et al., 2012). One of the important advantages of SILAC over other stable isotope labeling methods is that the label is integrated into the peptide at early stages of experimentation when the sample is metabolically active. Thus, possible variability due to sample preparation and purification losses are eliminated (Chahrour et al., 2015).

In this analysis, planktonic cells of GC strain 1291, which is an arginine auxotroph, were grown with labeled ${ }^{13} \mathrm{C}_{6}$-Arg, and biofilm cells were grown with unlabeled Arg in a continuousflow apparatus. Extracted protein samples from each bacterial population were combined and subjected to MALDI-TOF mass spectrometry analysis. Overall, this global analysis identified 757 proteins, 152 of which were significantly differentially expressed. In particular, GC cultured in a biofilm exhibited 73 upregulatedand 54 downregulated-proteins when compared to planktonic growth. The results of this study indicated that the bacteria upregulate proteins to respond to an oxygen-limited environment, including cytochrome $\mathrm{c}$ oxidase subunit III CcoP and nitrite reductase AniA. To cope with restricted nutrient availability in the biofilm, bacterial metabolism is shifted to increase sugar fermentation and tricarboxylic acid (TCA) cycle enzymes. The composition of the outer membrane is also altered during growth in a biofilm, with increased levels of 9 proteins including OpaB and OpaD (Phillips et al., 2012), both of which have been shown to adhere to and damage fallopian tube mucosa (Dekker et al., 1990). Among the downregulated proteins in the biofilm were proteins involved in energy metabolism, protein fate and synthesis, and transport and binding proteins, specifically iron complex outer membrane receptor protein (FetA) as well as transferrin-binding protein $\mathrm{B}$ and $\mathrm{A}$ (TbpB and TbpA, respectively).

A direct comparison of the transcriptome (Falsetta et al., 2009) and proteome expression profiles of GC biofilms showed a very poor correlation with only seven overlapping hits including AniA, OpaB, cytochrome C peroxidase CcpR, putative 
dihydrolipoamide dehydrogenase, putative cysteine synthase/ cystathionine beta-synthase, hypothetical protein NGO0905, and putative ABC transporter NGO1494 (Phillips et al., 2012).

This study gives insight into the adaptations necessary for GC to establish long-term infections and emphasizes the utility of proteomic approaches to examine these adaptations. In addition, the identified upregulated outer membrane proteins may be utilized as biomarkers for gonorrhea diagnostics.

\section{WHAT BOOK-LIKE CANDIDATE WAS NOT AT THE LAST PRESIDENTIAL DEBATE?}

\section{A NOVEL VACCINE CANDIDATE}

Perhaps one of the most exciting uses of proteomic approaches is in the search for new ways to combat multidrug resistant GC. We are applying a proteomics-driven reverse vaccinology approach to identify vaccine candidate proteins against gonorrhea (Zielke et al., 2014, 2015). Reverse vaccinology searches for possible vaccine candidate proteins using different genomics and proteomics methodologies and has already been successfully applied to different pathogenic bacteria including $N$. meningitidis serogroup B (Heckels and Williams, 2010; Adamczyk-Poplawska et al., 2011; Seib et al., 2012; Delany et al., 2013; Heinson et al., 2015).

No vaccine against GC currently exists, although research has been ongoing for decades. Two attempted vaccines, comprised of killed whole cells and purified pilin protein, failed in clinical trials over 13 years ago (Zhu et al., 2011). Since that time, very little research into gonorrheal vaccines has occurred, mainly due to the highly variable targeted surface proteins. Because GC is a strict human pathogen, research was also hampered by the lack of a suitable small animal model for gonorrheal infection (Jerse et al., 2014). Fortunately, a mouse model of female infection was developed, in which female mice are treated with $17-\beta$-estradiol when they are in the diestrus stage of the estrus cycle. The mice are also treated with an antibiotic cocktail of streptomycin sulfate, vancomycin $\mathrm{HCl}$, and trimethoprim sulfate to prevent overgrowth of commensal vaginal bacteria while under the influence of estradiol. 2 days after estradiol treatment, GC is introduced intravaginally. Using this model, GC can be recovered an average of 12.2 days post-inoculation with $10^{6}$ colony forming units (Jerse, 1999). A further advancement in the mouse model has been the development of transgenic mice that express human CEACAM proteins, providing a closer reproduction of conditions encountered in the human host (Jerse et al., 2014). The availability of a mouse model has greatly facilitated vaccine research. The immune response to infection, as well as resistance to subsequent infections after inoculation with an experimental vaccine can be closely monitored and investigated with the genetic tools available for studying mice (Zhu et al., 2011). However, to fully utilize this model for vaccine research, suitable candidate proteins must be identified-a goal for which proteomic approaches are ideally suited.

During the development of the MenB vaccine, out of nearly 600 candidates selected by reverse vaccinology, 350 recombinant proteins were successfully expressed in Escherichia coli and evaluated for their surface exposure. A total of 28 among them elicited bactericidal antibodies against Group B meningococci in vitro. Finally, the neisserial heparin-binding antigen NHBA, factor H-binding protein $\mathrm{fHbp}$, as well as the neisserial adhesin NadA were chosen as part of the MenB vaccine (Seib et al., 2012; Delany et al., 2013; Jerse et al., 2014). In contrast, only 12 different candidates are being evaluated as potential gonorrhea vaccine antigens (Jerse et al., 2014). Therefore, a more far-reaching effort is required to make a gonorrhea vaccine a reality.

Of particular interest for vaccine development and identification of new drug targets are proteins localized to the bacterial cell envelope and membrane vesicles (MVs) - spherical outpouchings of the cell envelope-as they interact directly or indirectly with host tissues; play roles in pathogenesis, antibiotic resistance, and biofilm formation; and participate in general physiological processes. Surprisingly few studies addressed GC cell envelope composition (Yoo et al., 2007; Phillips et al., 2012; Zielke et al., 2014). Also, despite studies reporting the release of MVs and their different morphological forms (spherical, lobed, and tubular) in GC from the early 1970s, only a few reports focused on elucidating their components (Swanson et al., 1971; Dorward et al., 1989; Pettit and Judd, 1992a,b; Falsetta et al., 2011; Zielke et al., 2014).

To begin the systematic mining of GC cell envelope and MVs for the discovery of vaccine and drug candidates, we first used the PSORTb 3.0.2 (Gardy et al., 2005) bioinformatics predictions and analyzed the subcellular localization of all ORFs in the completed genome sequence of strains FA1090 (Gen Bank accession number AE004969) and NCCP11945 (Gen Bank accession number CP001050), as well as the draft genome sequences of 14 different GC strains (downloaded from the Broad Institute website ${ }^{3}$ ). These studies revealed that, on average, about 50 of the 2,000 ORFs present in the GC genome encode outer membrane proteins (Zielke and Sikora, 2014). However, the subcellular location could not be predicted for about $30 \%$ of all ORFs. This analysis demonstrated that there is still much to learn about GC cell envelope composition and opened up exciting prospects for applying proteomics for the discovery of vaccine targets.

As proteomic investigations of membrane proteins are technically challenging, we chose to apply gel-free quantitative proteomic approaches including isobaric tagging for relative and absolute quantification (iTRAQ, Table 1) combined with multidimensional liquid chromatography and tandem mass spectrometry (2D-LC/MS/MS) to examine cell envelopes and naturally released MVs (Zielke et al., 2014). Four GC strains: FA1090, F62, MS11, and 1291 were cultured in liquid media under standard growth conditions and their cell envelopes and MVs were harvested in mid-logarithmic phase of growth. iTRAQ quantification was performed by labeling proteins isolated from subproteome fractions of each strain of interest with one of four isobaric tags (N-hydroxysuccinimide ester-activated compounds) that react to free amine groups on the N-termini and lysine side chains of proteins with high efficiency (Ross et al., 2004). Each of

${ }^{3} \mathrm{http} / /$ www.broadinstitute.org/annotation/genome/neisseria_gonorrhoeae/ MultiHome.html 
the four tags contains a reporter ion of a unique mass (Figure 1D; Table 1). When the samples are combined and subjected to mass spectrometry, the reporter ions are released from the labeled peptide. After the release of the reporter ions, all of the identical peptides in a sample will result in identical mass spectra, and the abundance of the peptide in the four multiplexed samples can be quantified by the relative intensity of the corresponding reporter ion peak (Ross et al., 2004; Wiese et al., 2007). The advantage of using iTRAQ is that it can be easily multiplexed and up to 8 different samples can be simultaneously analyzed within a single experiment. Additionally, as the iTRAQ tags react with all primary amine functional groups of peptides, nearly all peptides are labeled and information about not only their abundance but also their modification(s) can be acquired (Chahrour et al., 2015).

Our proteomic profiling of cell envelopes and native MVs revealed 533 and 168 common proteins, respectively, in analyzed GC strains. A total of 22 differentially abundant proteins were discovered including hitherto unknown proteins. Among those proteins that displayed similar abundance in four GC strains, we identified 305 and 46 cell envelope-and MVs-associated proteins, respectively. In addition, 34 proteins were found in both cell envelopes and MVs with eleven of them differentially regulated (Zielke et al., 2014). A few of these differentially expressed proteins included cytoplasmic proteins, an observation that was confirmed by a subsequent, independent proteomic study of MVs (Perez-Cruz et al., 2015).

The ubiquitous outer membrane proteins identified included GC homologs of the outer membrane $\beta$-barrel assembly (Bam) protein complex (Ricci and Silhavy, 2012), including BamA, BamD, and BamE; lipopolysaccharide transport protein LptD; and TamA (NGO1956) and TamB (NGO1955), two proteins thought to work cooperatively to assist in the assembly of autotransporter proteins (Heinz et al., 2015). Numerous uncharacterized proteins were also ubiquitously expressed, including NGO1344, NGO1985, NGO2111, NGO2121, and NGO2139 (Zielke et al., 2014).

We further examined LptD, NGO1344, NGO1985, NGO2111, NGO2121, NGO2139, and TamB by constructing conditional(LptD) or complete- knockout strains for each protein. These proteins were chosen because they contain domains predicted to function in maintaining cell envelope homeostasis. LptD expression in GC was placed under the control of an isopropyl $\beta$-D-1-thiogalactopyranoside (IPTG)-inducible promoter. This strain was unable to grow when streaked onto media lacking IPTG, while it grew robustly on plates supplemented with IPTG. Further, after $3 \mathrm{~h}$ of culturing in liquid media without IPTG, the bacteria ceased to grow. By $5 \mathrm{~h}$, bacterial viability decreased dramatically (nearly 13-fold) compared to the LptD-expressing strain. These experiments indicated that LptD is likely essential for GC viability. To test whether NGO1344, NGO1985, NGO2111, NGO2121, NGO2139, and TamB play functions in the integrity of the GC cell envelope, the individual clean deletion mutants were constructed in strain FA1090 and spotted on plates supplemented with various compounds. Although the loss of these proteins had no effect on bacterial growth under permissive conditions, the loss of NGO1985 resulted in a severe growth defect in the presence of bile salts, polymyxin B, Tween 20, SDS, urea, and chloramphenicol. Further, these phenotypes could be completely reversed by complementation with an IPTG-inducible version of the ngo1985 gene. Additionally, NGO2121 exhibited reduced growth in the presence of bile salts and polymyxin B. These proteins, identified by quantitative proteomic approaches, appear to provide an important function in maintaining cell membrane integrity and, as such, are promising targets for development of new therapeutic interventions against GC (Zielke et al., 2014).

To continue this research endeavor, we went on to determine the ubiquitously and specifically expressed cell envelope proteins of GC FA1090 challenged with host-relevant environmental stimuli: oxygen availability, iron deprivation, and the presence of human serum (Zielke et al., 2015). A myriad of novel proteins have been identified. Our initial characterization of five novel vaccine candidates that were ubiquitously expressed under these different growth conditions demonstrated that BamA, LptD, TamA, NGO2054, and NGO2139 were surface exposed and produced bactericidal antibodies that cross-reacted with a panel of diverse GC isolates. These promising results strongly suggest that the proteomics-driven approach will provide a foundation for the development of anti-GC vaccine(s), which would be the ideal way to prevent gonorrhea. Finally, to promote the utilization of the newly identified proteins and the knowledge of the GC subproteome dynamics among the scientific community, our entire data sets from all these investigations were made publicly available via the ProteomeXchange Consortium ${ }^{4}$, the PRIDE partner repository ProteomeXchange with the identifiers PXD000549 and PXD001944.

\section{FUTURE DIRECTIONS}

Although proteomic approaches have revealed a multitude of information on the physiology of GC, to formulate an effective vaccine, more information needs to be gathered about the way its surface proteins interact with host cells during an infection. Ideally, the proteome of clinical samples that have never been subcultured should be examined to determine which proteins are expressed during different stages of infection. This approach presents a significant technical challenge to overcome, as sufficient bacterial material must be collected for a quantitative proteomic analysis. In our investigation of the cell envelope and MV proteome, we collected material from 1 liter of culture (Zielke et al., 2014). Although other proteomic studies have used less material, including 5 milliliters of culture (Wu et al., 2010) or bacteria harvested from 20 plates (Anonsen et al., 2012; PerezCruz et al., 2015) collecting this amount of sample from a patient is not feasible; more sensitive MS analyzers or alternate methods of sample enrichment will be required. These studies will give insight into the proteomic adaptations the bacteria undergo to establish and maintain infection. Information collected from proteomic studies of clinical samples and host tissue culture can help further drive vaccine development and have the potential to aid in the discovery of stably expressed protein targets of antimicrobial agents with novel modes of action. Integration of multiple approaches, including public access to on-line raw data

\footnotetext{
${ }^{4}$ http://www.proteomexchange.org
} 
is essential if there is to be a sense of participation across the biomedical research community.

An interesting analysis technique was recently pioneered by Altindis et al. (2015). Termed "protectome analysis," their technique searches for vaccine candidate proteins in proteomic datasets by identifying proteins with structural or functional features in common with proteins known to provide protection. This analysis tool could be used in combination with other proteomic studies to immediately identify proteins expressed during infection with the potential to provide protection against reinfection.

As more information is deposited into proteomic databases, the utility of proteomic approaches to identify GC in clinical samples will increase. For example, in cases where the molecular determinant of antimicrobial resistance is known, proteomic approaches have the potential to immediately recognize the protein modification(s) that result in antimicrobial resistance, a feat that is not possible with current NAAT identification techniques.

Finally, proteomic investigations of multidrug resistance strains can reveal the mode of drug action, as well as the pathway(s) the bacterium uses to resist multiple antibiotics. One mystery that proteomic approaches may be able to solve is the identity of "Factor X," an unknown determinant of penicillin and cephalosporin resistance that is non-transformable and therefore is difficult to study with typical genetic methods (Unemo and Shafer, 2014).

\section{REFERENCES}

Adamczyk-Poplawska, M., Markowicz, S., and Jagusztyn-Krynicka, E. K. (2011). Proteomics for development of vaccine. J. Proteom. 74, 2596-2616. doi: 10.1016/j.jprot.2011.01.019

Altindis, E., Cozzi, R., Di Palo, B., Necchi, F., Mishra, R. P., Fontana, M. R., et al. (2015). Protectome analysis: a new selective bioinformatics tool for bacterial vaccine candidate discovery. Mol. Cell. Proteom. 14, 418-429. doi: 10.1074/mcp.M114.039362

Amiri-Eliasi, B., and Fenselau, C. (2001). Characterization of protein biomarkers desorbed by MALDI from whole fungal cells. Anal. Chem. 73, 5228-5231. doi: 10.1021/ac010651t

Anonsen, J. H., Egge-Jacobsen, W., Aas, F. E., Borud, B., Koomey, M., and Vik, A. (2012). Novel protein substrates of the phospho-form modification system in Neisseria gonorrhoeae and their connection to O-linked protein glycosylation. Infect. Immun. 80, 22-30. doi: 10.1128/IAI.05920-11

Bala, M., Kakran, M., Singh, V., Sood, S., Ramesh, V., and Members of WHO GASP SEAR Network. (2013). Monitoring antimicrobial resistance in Neisseria gonorrhoeae in selected countries of the WHO South-East Asia Region between 2009 and 2012: a retrospective analysis. Sex Transm. Infect. 89(Suppl. 4), iv28-iv35. doi: 10.1136/sextrans-2012-050904

Bilgin, N., Richter, A. A., Ehrenberg, M., Dahlberg, A. E., and Kurland, C. G. (1990). Ribosomal RNA and protein mutants resistant to spectinomycin. EMBO J. 9, 735-739.

Carannante, A., De Carolis, E., Vacca, P., Vella, A., Vocale, C., De Francesco, M. A., et al. (2015). Evaluation of matrix-assisted laser desorption ionization-time of flight mass spectrometry (MALDI-TOF MS) for identification and clustering of Neisseria gonorrhoeae. BMC Microbiol. 15:142. doi: 10.1186/s12866-015$0480-y$

CDC. (2012a). Cephalosporin-resistant Neisseria gonorrhoeae public health response plan. Available at: http://www.cdc.gov/std/treatment/Ceph-RResponsePlanJuly30-2012.pdf (accessed May 5, 2015).

CDC. (2012b). Update to CDC's Sexually transmitted diseases treatment guidelines, 2010: oral cephalosporins no longer a recommended treatment for

\section{CONCLUSION}

Developing novel vaccines or antimicrobial agents is critical in the face of growing antibiotic resistance, and global and quantitative proteomic approaches have begun to reveal potential targets in the fight against GC. Proteomic approaches are ideal for the discovery of vaccine candidate proteins, as well as protein targets for the development of novel antimicrobial agents. Qualitative proteomic studies revealed the GC defense response to spectinomycin, while quantitative proteomics have demonstrated bacterial adaptations to conditions encountered in the host, including oxidative stress, anoxia, iron deprivation, and the presence of human serum. Proteomics have also been recently adapted to identify GC in clinical samples, which can expedite treatment. Importantly for vaccine development, stably expressed proteins have been identified through high-throughput examinations of cell envelopes and naturally released MVs. These proteomic studies will act as starting points for studies into structural vaccinology, protein-protein interactions, and GC physiology, and have already given new insights into ways to combat this important, difficult to treat pathogen.

\section{ACKNOWLEDGMENTS}

Funding for this work was provided to AES by grant R01AI117235 from the National Institute of Allergy and Infectious Diseases, National Institutes of Health.

gonococcal infections. MMWR Morb. Mortal. Wkly. Rep. 61, 590-594. Available at: http://www.ncbi.nlm.nih.gov/pubmed/22874837 (accessed August 24, 2015).

CDC. (2013). 2013 STD Surveillance Gonorrhea. Available at: http://www. cdc.gov/std/stats13/gonorrhea.htm (accessed August 24, 2015).

Chahrour, O., Cobice, D., and Malone, J. (2015). Stable isotope labelling methods in mass spectrometry-based quantitative proteomics. J. Pharm. Biomed. Anal. 113, 2-20. doi: 10.1016/j.jpba.2015.04.013

Colangelo, C. M., and Williams, K. R. (2006). Isotope-coded affinity tags for protein quantification. Methods Mol. Biol. 328, 151-158. doi: 10.1385/1-59745-026$\mathrm{x}: 151$

Craig, A. P., Gray, R. T., Edwards, J. L., Apicella, M. A., Jennings, M. P., Wilson, D. P., et al. (2015). The potential impact of vaccination on the prevalence of gonorrhea. Vaccine 33, 4520-4525. doi: 10.1016/j.vaccine.2015. 07.015

Creighton, S. (2011). Gonorrhoea. BMJ Clin. Evid. 1604, 1-11.

Criss, A. K., Katz, B. Z., and Seifert, H. S. (2009). Resistance of Neisseria gonorrhoeae to non-oxidative killing by adherent human polymorphonuclear leucocytes. Cell. Microbiol. 11, 1074-1087. doi: 10.1111/j.1462-5822.2009. 01308.x

Dekker, N. P., Lammel, C. J., Mandrell, R. E., and Brooks, G. F. (1990). Opa (protein II) influences gonococcal organization in colonies, surface appearance, size and attachment to human fallopian tube tissues. Microb. Pathog. 9, 19-31. doi: 10.1016/0882-4010(90)90037-Q

Delany, I., Rappuoli, R., and Seib, K. L. (2013). Vaccines, reverse vaccinology, and bacterial pathogenesis. Cold Spring Harb. Perspect. Med. 3, a012476. doi: 10.1101/cshperspect.a012476

Dorward, D. W., Garon, C. F., and Judd, R. C. (1989). Export and intercellular transfer of DNA via membrane blebs of Neisseria gonorrhoeae. J. Bacteriol. 171, 2499-2505.

Ducey, T. F., Carson, M. B., Orvis, J., Stintzi, A. P., and Dyer, D. W. (2005). Identification of the iron-responsive genes of Neisseria gonorrhoeae by microarray analysis in defined medium. J. Bacteriol. 187, 4865-4874. doi: 10.1128/JB.187.14.4865-4874.2005 
ECDC. (2012). Response plan to control and manage the threat of multidrugresistant gonorrhoea in Europe. Available at: www.ecdc.europa.eu/en/ publications/ (accessed May 5, 2015).

Fagerquist, C. K., Garbus, B. R., Miller, W. G., Williams, K. E., Yee, E., Bates, A. H., et al. (2010). Rapid identification of protein biomarkers of Escherichia coli O157:H7 by matrix-assisted laser desorption ionization-time-of-flight-time-offlight mass spectrometry and top-down proteomics. Anal. Chem. 82, 2717-2725. doi: $10.1021 /$ ac $902455 \mathrm{~d}$

Falsetta, M. L., Bair, T. B., Ku, S. C., Vanden Hoven, R. N., Steichen, C. T., McEwan, A. G., et al. (2009). Transcriptional profiling identifies the metabolic phenotype of gonococcal biofilms. Infect. Immun. 77, 3522-3532. doi: 10.1128/IAI. 00036-09

Falsetta, M. L., Steichen, C. T., McEwan, A. G., Cho, C., Ketterer, M., Shao, J., et al. (2011). The composition and metabolic phenotype of Neisseria gonorrhoeae biofilms. Front. Microbiol. 2:75. doi: 10.3389/fmicb.2011.00075

Farley, T. A., Cohen, D. A., and Elkins, W. (2003). Asymptomatic sexually transmitted diseases: the case for screening. Prev. Med. 36, 502-509. doi: 10.1016/S0091-7435(02)00058-0

Fenselau, C., and Demirev, P. A. (2001). Characterization of intact microorganisms by MALDI mass spectrometry. Mass Spectrom. Rev. 20, 157-171. doi: 10.1002/mas.10004

Flemming, H. C., and Wingender, J. (2010). The biofilm matrix. Nat. Rev. Microbiol. 8, 623-633. doi: $10.1038 /$ nrmicro2415

Galimand, M., Gerbaud, G., and Courvalin, P. (2000). Spectinomycin resistance in Neisseria spp. due to mutations in $16 \mathrm{~S}$ rRNA. Antimicrob. Agents Chemother. 44, 1365-1366. doi: 10.1128/AAC.44.5.1365-1366.2000

Gardy, J. L., Laird, M. R., Chen, F., Rey, S., Walsh, C. J., Ester, M., et al. (2005). PSORTb v.2.0: expanded prediction of bacterial protein subcellular localization and insights gained from comparative proteome analysis. Bioinformatics 21, 617-623. doi: 10.1093/bioinformatics/bti057

Greiner, L. L., Edwards, J. L., Shao, J., Rabinak, C., Entz, D., and Apicella, M. A. (2005). Biofilm formation by Neisseria gonorrhoeae. Infect. Immun. 73, 1964-1970. doi: 10.1128/IAI.73.4.1964-1970.2005

Gudlavalleti, S. K., Sundaram, A. K., Razumovski, J., and Doroshenko, V. (2008). Application of atmospheric pressure matrix-assisted laser desorption/ionization mass spectrometry for rapid identification of Neisseria species. J. Biomol. Tech. $19,200-204$

Gygi, S. P., Rist, B., Gerber, S. A., Turecek, F., Gelb, M. H., and Aebersold, R. (1999). Quantitative analysis of complex protein mixtures using isotope-coded affinity tags. Nat. Biotechnol. 17, 994-999. doi: 10.1038/13690

Heckels, J. E., and Williams, J. N. (2010). The influence of genomics and proteomics on the development of potential vaccines against meningococcal infection. Genom. Med. 2, 43. doi: $10.1186 / \mathrm{gm} 164$

Heinson, A. I., Woelk, C. H., and Newell, M. L. (2015). The promise of reverse vaccinology. Int. Health 7, 85-89. doi: 10.1093/inthealth/ihv002

Heinz, E., Selkrig, J., Belousoff, M. J., and Lithgow, T. (2015). Evolution of the translocation and assembly module (TAM). Genome Biol. Evol. 7, 1628-1643. doi: 10.1093/gbe/evv097

Ilina, E. N., Borovskaya, A. D., Malakhova, M. M., Vereshchagin, V. A., Kubanova, A. A., Kruglov, A. N., et al. (2009). Direct bacterial profiling by matrix-assisted laser desorption-ionization time-of-flight mass spectrometry for identification of pathogenic Neisseria. J. Mol. Diagn. 11, 75-86. doi: 10.2353/jmoldx.2009.080079

Ilina, E. N., Oparina, N. Y., Shitikov, E. A., Borovskaya, A. D., and Govorun, V. M. (2010). Molecular surveillance of clinical Neisseria gonorrhoeae isolates in Russia. J. Clin. Microbiol. 48, 3681-3689. doi: 10.1128/JCM. 00565-10

Isabella, V. M., and Clark, V. L. (2011). Deep sequencing-based analysis of the anaerobic stimulon in Neisseria gonorrhoeae. BMC Genom. 12:51. doi: $10.1186 / 1471-2164-12-51$

Jerse, A. E. (1999). Experimental gonococcal genital tract infection and opacity protein expression in estradiol-treated mice. Infect. Immun. 67, 5699-5708.

Jerse, A. E., Bash, M. C., and Russell, M. W. (2014). Vaccines against gonorrhea: current status and future challenges. Vaccine 32, 1579-1587. doi: 10.1016/j.vaccine.2013.08.067

Low, N., Unemo, M., Skov Jensen, J., Breuer, J., and Stephenson, J.M. (2014). Molecular diagnostics for gonorrhoea: implications for antimicrobial resistance and the threat of untreatable gonorrhoea. PLoS Med. 11:e1001598. doi: 10.1371/journal.pmed.1001598
Maness, M. J., Foster, G. C., and Sparling, P. F. (1974). Ribosomal resistance to streptomycin and spectinomycin in Neisseria gonorrhoeae. J. Bacteriol. 120, 1293-1299.

McClure, R., Nudel, K., Massari, P., Tjaden, B., Su, X., Rice, P. A., et al. (2015) The Gonococcal Transcriptome during Infection of the Lower Genital Tract in Women. PLoS ONE 10:e0133982. doi: 10.1371/journal.pone.0133982

Murray, P. R. (2010). Matrix-assisted laser desorption ionization time-of-flight mass spectrometry: usefulness for taxonomy and epidemiology. Clin. Microbiol. Infect. 16, 1626-1630. doi: 10.1111/j.1469-0691.2010.03364.x

Nabu, S., Lawung, R., Isarankura-Na-Ayudhya, P., Isarankura-Na-Ayudhya, C., Roytrakul, S., and Prachayasittikul, V. (2014). Reference map and comparative proteomic analysis of Neisseria gonorrhoeae displaying high resistance against spectinomycin. J. Med. Microbiol. 63, 371-385. doi: 10.1099/jmm.0.067595-0

Niyompanich, S., Jaresitthikunchai, J., Srisanga, K., Roytrakul, S., and Tungpradabkul, S. (2014). Source-identifying biomarker ions between environmental and clinical Burkholderia pseudomallei using whole-cell matrix-assisted laser desorption/ionization time-of-flight mass spectrometry (MALDI-TOF MS). PLoS ONE 9:e99160. doi: 10.1371/journal.pone.0099160

Otto, A., Bernhardt, J., Hecker, M., and Becher, D. (2012). Global relative and absolute quantitation in microbial proteomics. Curr. Opin. Microbiol. 15, 364-372. doi: 10.1016/j.mib.2012.02.005

Perez-Cruz, C., Delgado, L., Lopez-Iglesias, C., and Mercade, E. (2015). Outer-inner membrane vesicles naturally secreted by gram-negative pathogenic bacteria. PLOS ONE 10:e0116896. doi: 10.1371/journal.pone.0116896

Pettit, R. K., and Judd, R. C. (1992a). Characterization of naturally elaborated blebs from serum-susceptible and serum-resistant strains of Neisseria gonorrhoeae. Mol. Microbiol. 6, 723-728. doi: 10.1111/j.1365-2958.1992.tb01521.x

Pettit, R. K., and Judd, R. C. (1992b). The interaction of naturally elaborated blebs from serum-susceptible and serum-resistant strains of Neisseria gonorrhoeae with normal human serum. Mol. Microbiol. 6, 729-734. doi: 10.1111/j.13652958.1992.tb01522.x

Phillips, N. J., Steichen, C. T., Schilling, B., Post, D. M., Niles, R. K., Bair, T. B., et al. (2012). Proteomic analysis of Neisseria gonorrhoeae biofilms shows shift to anaerobic respiration and changes in nutrient transport and outer membrane proteins. PLoS ONE 7:e38303. doi: 10.1371/journal.pone.0038303

Ramakrishnan, V., and White, S. W. (1992). The structure of ribosomal protein S5 reveals sites of interaction with $16 \mathrm{~S}$ rRNA. Nature 358, 768-771. doi: $10.1038 / 358768 \mathrm{a} 0$

Ricci, D. P., and Silhavy, T. J. (2012). The Bam machine: a molecular cooper. Biochim. Biophys. Acta 1818, 1067-1084. doi: 10.1016/j.bbamem.2011.08.020

Ross, P. L., Huang, Y. N., Marchese, J. N., Williamson, B., Parker, K., Hattan, S., et al. (2004). Multiplexed protein quantitation in Saccharomyces cerevisiae using amine-reactive isobaric tagging reagents. Mol. Cell. Proteomics 3, 1154-1169. doi: 10.1074/mcp.M400129-MCP200

Sarantis, H., and Gray-Owen, S. D. (2012). Defining the roles of human carcinoembryonic antigen-related cellular adhesion molecules during neutrophil responses to Neisseria gonorrhoeae. Infect. Immun. 80, 345-358. doi: 10.1128/IAI.05702-11

Schmid, O., Ball, G., Lancashire, L., Culak, R., and Shah, H. (2005). New approaches to identification of bacterial pathogens by surface enhanced laser desorption/ionization time of flight mass spectrometry in concert with artificial neural networks, with special reference to Neisseria gonorrhoeae. J. Med. Microbiol. 54, 1205-1211. doi: 10.1099/jmm.0.46223-0

Schmitter, T., Pils, S., Weibel, S., Agerer, F., Peterson, L., Buntru, A., et al. (2007). Opa proteins of pathogenic neisseriae initiate Src kinase-dependent or lipid raft-mediated uptake via distinct human carcinoembryonic antigenrelated cell adhesion molecule isoforms. Infect. Immun. 75, 4116-4126. doi: 10.1128/IAI.01835-06

Seib, K. L., Tseng, H. J., McEwan, A. G., Apicella, M. A., and Jennings, M. P. (2004). Defenses against oxidative stress in Neisseria gonorrhoeae and Neisseria meningitidis: distinctive systems for different lifestyles. J. Infect. Dis. 190, 136-147. doi: 10.1086/421299

Seib, K. L., Wu, H. J., Kidd, S. P., Apicella, M. A., Jennings, M. P., and McEwan, A. G. (2006). Defenses against oxidative stress in Neisseria gonorrhoeae: a system tailored for a challenging environment. Microbiol. Mol. Biol. Rev. 70, 344-361. doi: 10.1128/MMBR.00044-05

Seib, K. L., Zhao, X., and Rappuoli, R. (2012). Developing vaccines in the era of genomics: a decade of reverse vaccinology. Clin. Microbiol. Infect. 18(Suppl. 5), 109-116. doi: 10.1111/j.1469-0691.2012.03939.x 
Simons, M. P., Nauseef, W. M., and Apicella, M. A. (2005). Interactions of Neisseria gonorrhoeae with adherent polymorphonuclear leukocytes. Infect. Immun. 73, 1971-1977. doi: 10.1128/IAI.73.4.1971-1977.2005

Sintsova, A., Sarantis, H., Islam, E. A., Sun, C. X., Amin, M., Chan, C. H., et al. (2014). Global analysis of neutrophil responses to Neisseria gonorrhoeae reveals a self-propagating inflammatory program. PLoS Pathog. 10:e1004341. doi: 10.1371/journal.ppat.1004341

Steichen, C. T., Shao, J. Q., Ketterer, M. R., and Apicella, M. A. (2008). Gonococcal cervicitis: a role for biofilm in pathogenesis. J. Infect. Dis. 198, 1856-1861. doi: $10.1086 / 593336$

Swanson, J., Kraus, S. J., and Gotschlich, E. C. (1971). Studies on gonococcus infection. I. Pili and zones of adhesion: their relation to gonococcal growth patterns. J. Exp. Med. 134, 886-906. doi: 10.1084/jem.134.4.886

Tapsall, J. W. (2005). Antibiotic resistance in Neisseria gonorrhoeae. Clin. Infect. Dis. 41(Suppl. 4), S263-S268. doi: 10.1086/430787

Tapsall, J. W., Ray, S., and Limnios, A. (2010). Characteristics and population dynamics of mosaic penA allele-containing Neisseria gonorrhoeae isolates collected in Sydney, Australia, in 2007-2008. Antimicrob. Agents Chemother. 54, 554-556. doi: 10.1128/AAC.01108-09

Tseng, H. J., Srikhanta, Y., McEwan, A. G., and Jennings, M. P. (2001). Accumulation of manganese in Neisseria gonorrhoeae correlates with resistance to oxidative killing by superoxide anion and is independent of superoxide dismutase activity. Mol. Microbiol. 40, 1175-1186. doi: 10.1046/j.1365-2958.2001.02460.x

Unemo, M. (2015). Current and future antimicrobial treatment of gonorrhoea-the rapidly evolving Neisseria gonorrhoeae continues to challenge. BMC Infect. Dis. 15:364. doi: 10.1186/s12879-015-1029-2

Unemo, M., Golparian, D., Skogen, V., Olsen, A. O., Moi, H., Syversen, G., et al. (2013). Neisseria gonorrhoeae strain with high-level resistance to spectinomycin due to a novel resistance mechanism (mutated ribosomal protein S5) verified in Norway. Antimicrob. Agents Chemother. 57, 1057-1061. doi: 10.1128/AAC.01775-12

Unemo, M., and Shafer, W. M. (2014). Antimicrobial resistance in Neisseria gonorrhoeae in the 21st century: past, evolution, and future. Clin. Microbiol. Rev. 27, 587-613. doi: 10.1128/CMR.00010-14

van Baar, B. L. (2000). Characterisation of bacteria by matrix-assisted laser desorption/ionisation and electrospray mass spectrometry. FEMS Microbiol. Rev. 24, 193-219. doi: 10.1016/S0168-6445(99)00036-4

Wiese, S., Reidegeld, K. A., Meyer, H. E., and Warscheid, B. (2007). Protein labeling by iTRAQ: a new tool for quantitative mass spectrometry in proteome research. Proteomics 7, 340-350. doi: 10.1002/pmic.200600422

Wind, C. M., de Vries, H. J., Schim van der Loeff, M. F., Unemo, M., and van Dam, A. P. (2015). Successful combination of nucleic acid amplification test diagnostics and targeted deferred Neisseria gonorrhoeae culture. J. Clin. Microbiol. 53, 1884-1890. doi: 10.1128/JCM.00369-15
WHO. (2011). Prevalence and incidence of selected sexually transmitted infections. Chlamydia trachomatis, Neisseria gonorrhoeae, syphilis, and Trichomonas vaginalis. Methods, and results used by WHO to generate 2005 Estimates. 36. Available at: http://www.who.int/reproductivehealth/ publications/rtis/9789241502450/en/ (accessed August 27, 2015).

WHO. (2012). Global action plan to control the spread and impact of antimicrobial resistance in Neisseria gonorrhoeae. Available at: http://whqlibdoc.who.int/publications/2012/9789241503501_eng.pdf (accessed May 5, 2015)

Wu, H. J., Seib, K. L., Srikhanta, Y. N., Edwards, J., Kidd, S. P., Maguire, T. L., et al. (2010). Manganese regulation of virulence factors and oxidative stress resistance in Neisseria gonorrhoeae. J. Proteom. 73, 899-916. doi: 10.1016/j.jprot.2009.12.001

Yoo, J. S., Seong, W. K., Kim, T. S., Park, Y. K., Oh, H. B., and Yoo, C. K. (2007). Comparative proteome analysis of the outer membrane proteins of in vitroinduced multi-drug resistant Neisseria gonorrhoeae. Microbiol. Immunol. 51, 1171-1177. doi: 10.1111/j.1348-0421.2007.tb04012.x

Zhu, W., Chen, C. J., Thomas, C. E., Anderson, J. E., Jerse, A. E., and Sparling, P. F. (2011). Vaccines for gonorrhea: can we rise to the challenge? Front. Microbiol. 2:124. doi: 10.3389/fmicb.2011.00124

Zielke, R. A., and Sikora, A. E. (2014). Isolation of cell envelopes and naturally released membrane vesicles of Neisseria gonorrhoeae. Curr. Protoc. Microbiol. 34, 4A 3 1-4A 3 17. doi: 10.1002/9780471729259.mc04 a03s34

Zielke, R. A., Wierzbicki, I. H., Jerse, A. E., Gafken, P. R., Soge, O. O., Holmes, K. K., et al. (2015). Proteomics-driven Reverse Vaccinology for Gonorrhea. Available at: www.abstractsonline.com/Plan/ViewAbstract.aspx?mID=3729 $\&$ sKey $=$ fa3e7acf-5429-438d-84f7-832cle0ed $4 b 4 \& c K e y=c 67 c 0637-58 c 5-4 e a 2-$ 9872-869ccc94ef34\&mKey=224bad71-94ea-4fa5-8df3-f4087bdc3625

Zielke, R. A., Wierzbicki, I. H., Weber, J. V., Gafken, P. R., and Sikora, A. E. (2014). Quantitative proteomics of the Neisseria gonorrhoeae cell envelope and membrane vesicles for the discovery of potential therapeutic targets. Mol. Cell Proteom. 13, 1299-1317. doi: 10.1074/mcp.M113.029538

Conflict of Interest Statement: The authors declare that the research was conducted in the absence of any commercial or financial relationships that could be construed as a potential conflict of interest.

Copyright (C) 2015 Baarda and Sikora. This is an open-access article distributed under the terms of the Creative Commons Attribution License (CC BY). The use, distribution or reproduction in other forums is permitted, provided the original author(s) or licensor are credited and that the original publication in this journal is cited, in accordance with accepted academic practice. No use, distribution or reproduction is permitted which does not comply with these terms. 